ARTIGOS

\section{SATISFAÇÃO NO TRABALHO: ANTECEDENTES E CONSEQUENTES}

\author{
JOB SATISFACTION: ANTECEDENTS AND \\ CONSEQUENTS
}

\section{RESUMO}

Este estudo pretende compreender as variáveis que promovem satisfação nos trabalhadores, contribuindo para um melhor entendimento do conceito, bem como para que as organizações compreendam que os seus recursos humanos, quando satisfeitos, tornam-se uma vantagem competitiva para alcançar o sucesso organizacional. Para verificar tais hipóteses, recorreu-se a um estudo cross-section, utilizando um questionário, em que se obtiveram 418 respostas de trabalhadores portugueses. Para testar as hipóteses, examinou-se o modelo das equações estruturais recorrendo ao software estatístico IBM SPSS AMOS v.25. Os resultados apontaram que as variáveis escolhidas como antecedentes, com exceção do reconhecimento, influenciam a satisfação de um trabalhador, pois, quando um trabalhador está satisfeito, não só ele está com a vida satisfeita, como há uma maior orientação para o cliente, que é mais comprometido e leal, visto que sente mais orgulho da organização e é dotado de uma maior iniciativa pessoal.

Palavras-chave: Satisfação no trabalho. Recursos humanos. Sucesso Organizacional. Vantagem competitiva.

\begin{abstract}
This study aims to understand the variables that promote employee satisfaction, contributing to a better understanding of the concept, as well as for organizations to understand that their human resources, when satisfied, become a competitive advantage to achieve organizational success. To verify such hypotheses a cross-sectional study was resorted using a questionnaire, in which 418 responses were obtained from Portuguese workers. To test the hypotheses, the structural equations model was examined using the IBM SPSS AMOS v.25 statistical software. The results pointed out that the variables chosen as antecedents, with the exception of recognition, influence the workers' satisfaction and that a contented worker is not only gratified with their lives but there is a greater orientation
\end{abstract}


towards the customers, who are more committed and loyal, since they feel more proud of the organization and are endowed with a greater personal initiative.

Keywords: Job Satisfaction. Human Resources. Organizational Success. Competitive advantage.

\section{INTRODUÇÃO}

Diante da dinamicidade dos mercados, diversos fatores como os aumentos exponenciais da concorrência tendem a gerar dificuldades para a obtenção de vantagens competitivas, maiores margens de lucro e, por conseguinte, o sucesso. Segundo diversos autores, como Sibhoko e Bayat (2019), a preocupação exclusiva com os consumidores se tornou insuficiente para garantir o maior sucesso e, assim, é reconhecida a importância dos trabalhadores como estratégia para uma organização ultrapassar os obstáculos e tornar-se bem-sucedida.

Nesse contexto, para uma organização se apoiar nos seus recursos humanos, é indispensável que o trabalhador se sinta satisfeito com o seu trabalho, pois só assim irá estender todos os esforços em suas tarefas para atingir os objetivos organizacionais e proporcionar um melhor atendimento ao cliente, que, ultimamente, trará lucro para a empresa (SIBHOKO; BAYAT, 2019). Desse modo, este artigo parte da ideia de que essa satisfação é fundamental para o crescimento e o sucesso das organizações, bem como para o desenvolvimento e sucesso profissional e pessoal dos trabalhadores.

A importância deste tema, que nasce nas corrente psicológicas, emergiu para as áreas de gestão, recursos humanos e marketing, pois já se aceita, hoje, com naturalidade, que trabalhadores satisfeitos representam uma estratégia inteligente para se superar obstáculos e ganhar uma posição competitiva favorável (LINDON et al., 2004), de modo que as empresas devem adotar uma perspetiva de marketing direcionada para motivar e satisfazer os trabalhadores (AHMED; RAFIQ, 2002).
Devido à consciencialização da importância do tema satisfação no trabalho, este foi largamente estudado ao longo dos anos; no entanto, segundo Pradhan, Dash e Jena (2019), devido à complexidade dos mercados, existem, ainda, algumas lacunas, nomeadamente, sobre o que leva a um trabalhador ficar satisfeito. Também, Alegre, Mas-Machuca e Berbegal-Mirabent (2016) encontram, como lacuna nesses estudos, o facto de as investigações se concentrarem em relações one-to-one com a variável satisfação no trabalho, sugerindo que estudos futuros incluam combinações de relacionamentos dentro do trabalho. Igualmente, Sibhoko e Bayat (2019), devido ao papel da temática no desempenho geral das organizações, defendem que essas futuras investigações deveriam explorar, em maior profundidade, a maneira com que um trabalhador satisfeito contribui para os resultados positivos organizacionais.

Assim, este artigo tem como objetivo contribuir para a contínua compreensão do tema, apresentando novas abordagens sobre ele, adequando-as às realidades mais recentes dos trabalhadores; perceber se as variáveis LMX, TMX, Reconhecimento, Segurança no Trabalho, Empowerment, Clima Organizacional, Stress no trabalho, Salário e o Equilíbrio Emocional e Paz Interior são antecedentes da satisfação nos trabalhadores e se, efetivamente, um trabalhador satisfeito apresenta uma maior Satisfação com a Vida, Orientação para o Cliente, Comprometimento Afetivo Organizacional, Lealdade, Orgulho na Organização e Iniciativa Pessoal.

Para verificar tais hipóteses, recorreu-se a um estudo cross-section, utilizando um questionário, alcançando 418 respostas. Este estudo diferencia-se dos demais, pois é desenvolvido a partir da examinação da bibliografia conceituada sobre o tema; sugere-se um agrupamento de variáveis que nunca foram estudadas, simultaneamente, em relação a esta temática. Igualmente, os estudos desenvolvidos, anteriormente, concentram-se meramente, na examinação dos antecedentes ou dos 
consequentes (PRAJOGO, 2019). Dessa forma, esta pesquisa, de uma maneira inovadora, pretende combinar a ambas.

\section{DESENVOLVIMENTO CONCEPTUAL E HIPÓTESES DE INVESTIGAÇÃO}

\subsection{SATISFAÇÃO NO TRABALHO}

Como abordado, é crucial haver trabalhadores satisfeitos e, apesar de este tema ter sido extensamente debatido, é necessária sua contínua compreensão com o intuito de aprofundá-la.

Historicamente, essa problemática foi explorada a partir de 1910, no cume do taylorismo e na indústria norte-americana. No entanto, na década de 1970, devido à crise mundial do petróleo, que obrigou as empresas a reajustamentos apressados na produção movida pela complexidade e incertezas dos mercados, a preocupação com os trabalhadores foi deixada para segundo plano, só sendo retomada, mais tarde, a consciência de que o fator humano é importante na superação dos obstáculos (CUNHA et al., 2012).

Devido à complexidade da temática, considera-se que compreendê-la não é tarefa fácil, pois pode ser estudada mediante várias abordagens (SIBHOKO; BAYAT, 2019). Assim, como exemplo, existe a teoria de Maslow (1943) que tenta explicar a temática por meio das necessidades humanas, que são apresentadas em uma pirâmide de cinco níveis, denominadas, hierarquicamente, em necessidades fisiológicas, de segurança, sociais, estima e autorrealização, ou a teoria de Herzberg, Mausner e Snyderman (1959) que a tentam explicar pela motivação, ou seja, tentaram descobrir quais os fatores que levavam à satisfação e quais os que levavam à insatisfação, dividindo-os em fatores de higiene, relacionando-os com o ambiente de trabalho, e que podem levar à insatisfação (i.e. política da organização, salários, supervisão, relações com os colegas, segurança e condições de trabalho) e os fatores motivacionais, como sendo os que realmente motivam e satisfazem os trabalhadores (i.e. reconhecimento, realização, promoção, crescimento, trabalho e responsabilidade).

Assim, como as teorias, a definição de satisfação também é muito debatida, de inúmeras formas por diversos autores. Locke (1969, p. 316, tradução nossa) define satisfação no trabalho como "[...] o estado emocional prazeroso resultante da avaliação do seu trabalho", Spector (1997, p. 2, tradução nossa) define-a como "à medida que as pessoas gostam (satisfação) ou não gostam (insatisfação) dos seus trabalhos" e Aristovnik et al. (2018, p. 129, tradução nossa) descrevem-na como uma "[...] sensação agradável que uma pessoa tem quando suas expectativas do trabalhado foram satisfeitas", enquanto Robbins e Coulter (1996) e Weiss (2002) consideram que a satisfação no trabalho refere-se às atitudes que os colaboradores têm perante suas funções e local de trabalho.

Nguyen, Taylor e Bradley (2003, p. 5, tradução nossa) interpretam a satisfação no trabalho como uma abordagem multidimensional, em que "a satisfação geral com o trabalho é determinada pela satisfação em vários domínios de trabalho, como remuneração, segurança no emprego, perspetivas de promoção, benefícios adicionais e a importância atribuída ao trabalho" enquanto Netemeyer et al. (1997) e Judge, Bono e Locke (2000) medem-na de forma unidimensional, ou seja, de forma geral e não medindo os vários domínios do trabalho.

No entanto, todos os autores consentem que a satisfação no trabalho é muito importante para o desenvolvimento das organizações, bem como para a evolução profissional e pessoal dos trabalhadores. Utilizar os recursos humanos da empresa como uma vantagem competitiva é uma prática de diferenciação inteligente, porém previamente. É preciso trabalhar a satisfação desses para que estejam dispostos a trabalhar em prol dos objetivos organizacionais, amplificando o sucesso da empresa (PRADHAN; DASH; JENA, 2019). Já Spector (1997) apontava que a importância dessa temática é, primeiramente, devido a uma perspetiva humana, pois as pesso- 
as merecem ser bem tratadas e sentem-se bem nos seus trabalhos, e, em segundo lugar, por uma perspetiva utilitarista, pois a satisfação poderá afetar o comportamento organizacional. Também essa importância é devido à forma como os trabalhadores encaram o posto de trabalho; pois, atualmente, procuram gostar realmente do que fazem, satisfazendo as expectativas de realização pessoal (CUNHA et al., 2012).

Dos inúmeros autores que se dedicaram a estudar a satisfação no trabalho, muitos apontam que existem diversos fatores inerentes às organizações que se podem traduzir em trabalhadores satisfeitos, nomeadamente, uns defendem que as remunerações, a autonomia dada aos trabalhadores e a segurança impactam a satisfação (SARKER; ASHRAFI, 2018) e, por outro lado, outros relacionam as condições e o ambiente de trabalho, o reconhecimento e os relacionamentos com os colegas e o com os líderes com um trabalhador satisfeito, pois acreditam que preenchem uma necessidade de interação social que é inata ao ser humano (SINHA; KUMAR, 2012). Ainda, outros autores afirmam que esta é de grande importância em nível organizacional, pessoal e profissional, pois suas pesquisas revelaram que existe um elo entre um trabalhador satisfeito, a sua lealdade e o comprometimento para com a organização, assim como um melhor relacionamento com os clientes afetando, também, positivamente, a satisfação com a sua própria vida (SARKER; ASHRAFI, 2018)

Pode-se, assim, confirmar que a satisfação no trabalho é de fato um tema de grande interesse e, por isso, serão abordados, em maior profundidade, alguns antecedentes e consequentes com o intuito de compreender melhor a sua ligação com a satisfação no trabalho.

\subsection{ANTECEDENTES DA SATISFA- ÇÃO NO TRABALHO}

Um bom relacionamento com o líder é fundamental para o bom funcionamento organizacional e bem-estar geral, pois os líderes têm a capacidade de influenciar o comportamento dos seus liderados, fazendo que se sintam bem e motivados a trabalhar em prol dos objetivos organizacionais (ALMEIDA, 2016).

Dessa forma, Leader-member exchange (LMX) diz respeito a uma teoria que recai na qualidade dos relacionamentos entre líderes e liderados, encerrados em uma tripla vertente, nomeadamente, respeito, confiança e obrigação (GRAEN; UHL-BIEN, 1995).

Quando os trabalhadores gozam de bons relacionamentos com seus líderes, tendo atenção e apoio deles (MORROW et al., 2005), sentem-se bem tratados e isso se reflete na forma como encaram seu trabalho, considerando-o como sendo mais satisfatório, reportando maiores níveis de satisfação com ele (BHAL; GULATI; ANSARI, 2009). Dessa forma, propõe-se a seguinte hipótese:

\section{H1: O LMX está relacionado positivamente com a Satisfação no Trabalho.}

Igualmente, um bom relacionamento com os colegas de trabalho é fundamental para o bem-estar individual e geral dentro de uma empresa, pois, além de os trabalhadores despenderem muitas horas, diariamente, interagindo com os colegas, cada vez mais, as organizações recorrem ao trabalho em equipa para resoluções de problemas e alcance de objetivos (MESMER-MAGNUS; DECHURCH, 2009).

Dessa forma, Team-member Exchange (TMX) refere-se a uma teoria, adaptada da teoria LMX, sobre a perceção do trabalhador da qualidade das relações entre os membros de uma equipe de trabalho (SEERS; PETTY; CASHMAN, 1995).

Consequentemente, quando um trabalhador goza de bons relacionamentos com a sua equipe, ou seja, existem reciprocidade, feedba$c k$ e entreajuda, consideram as suas experiências no trabalho satisfatórias (SEERS; PETTY; CASHMAN, 1995), reportando, assim, a uma maior satisfação com ele (BANKS et al., 2014). Consequentemente, propõe-se a seguinte hipótese:

H2: O TMX está relacionado, positivamente, 


\section{com a Satisfação no Trabalho.}

Como os trabalhadores contribuem para o desenvolvimento das organizações, recompensá-los e reconhecê-los é fundamental para que se sintam motivados a trabalhar mais e melhor (ALBALOOSHI; ALI; AL-ANSI, 2017).

Para este estudo, adotou-se que reconhecimento é “[...] uma expressão pública de apreciação dada por um grupo a indivíduos que assumem comportamentos desejados" (FISHER; ACKERMAN, 1998, p. 264, tradução nossa) que é "[...] dado frequentemente em conjunto com recompensas tangíveis" (YUKL; GORDON; TABER, 2002, p. 21, tradução nossa).

Apesar de o reconhecimento ter sido alvo de pouca atenção por parte dos autores como um fator que leve à satisfação de um trabalhador, já Maslow (1943) o considerava como uma necessidade básica dos indivíduos, segundo Shen e Tang (2018) afirmaram, no seu estudo, o reconhecimento dado aos trabalhadores é deveras importante porque eles, quando são distinguidos pelo bom desempenho, sentem-se valorizados pelo seu esforço desenvolvendo uma maior satisfação com o seu trabalho (ALIAS et al., 2018). Dessa forma, propõe-se a seguinte hipótese:

\section{H3: O Reconhecimento está relacionado po-} sitivamente com a Satisfação no Trabalho.

Devido às dificuldades econômicas combinadas com mudanças drásticas na natureza do trabalho, nomeadamente o downsizing, corte de custos, despedimentos e redução de contratos efetivos, a incerteza dos trabalhadores em relação a seus postos de trabalho aumentou (JIANG; PROBST, 2016)this article research. (PsycINFO Database Record (c.

Por segurança, considera-se a definição de Davy, Kinicki e Scheck (1997, p. 323, tradução nossa) a qual é vista como sendo as " [...] expectativas de uma pessoa sobre a continuidade numa situação de trabalho" em que é dado aos trabalhadores a "[...] garantia razoável de que eles não serão demitidos, mesmo durante ciclos econômicos difíceis." (BANSAL; MENDELSON; SHARMA, 2001, p. 66, tradução nossa)organizations must focus their efforts on developiemployees (longitudinal $n=906$, consideram que é crucial que uma empresa garanta a segurança dos seus trabalhadores, pois não só recebe, em troca, desempenho e lealdade, como também fará que o trabalhador reporte maiores níveis de satisfação relativamente a seu traba1ho. Pelo exposto, propõe-se a seguinte hipótese:

\section{H4: A Segurança está relacionada, positiva-} mente, com a Satisfação no Trabalho.

Devido à instabilidade no mercado, as empresas recorrem ao conhecimento, às ideias e à criatividade de seus recursos humanos, como uma forma de vantagem competitiva sustentável (YIN; WANG; LU, 2018). Para que os trabalhadores possam contribuir para o sucesso organizacional, é necessário que as organizações os envolvam nas tomadas de decisões, dando-lhes empowerment (YIN; WANG; LU, 2018), que, de acordo com Gounaris (2008)the extant literature reports that the number of companies practicing marketing internally is disproportionate small compared to the number of companies trying to adopt the market orientation concept Hence, the purpose of this paper is to offer a preliminary insight regarding the antecedents of practicing marketing internally. Design/methodology/approach - To do this, data were collected from 583 first-line personnel from 29 five and four stars hotels in Greece through personal interviews in order to investigate the impact of company culture and internal-market orientation (IMO, refere-se à permissão e ao poder dado aos trabalhadores para que tomem decisões sobre o seu trabalho.

Consequentemente, essa maior autonomia e capacidade de decisão dada aos trabalhadores produzem resultados positivos em suas atitudes e comportamentos; pois, efetivamente, possuem um maior controlo sobre suas tarefas, têm maior flexibilidade em tomar decisões e sentem-se honrados pela confiança depositada, o que aumenta a sua confiança e o sentimento de satisfação ao realizar o seu trabalho (KIM; FERNANDEZ, 2017)gerial approach on turnover options in the public sector. This study proposes a theoretical model of the direct 
and indirect effects of employee empowerment on turnover intention in the U.S. federal bureaucracy. The model is tested using structural equation modeling (SEM. Por conseguinte, propõe-se a seguinte hipótese:

H5: O Empowerment está relacionado, positivamente, com a Satisfação no Trabalho.

As perceções que os trabalhadores têm sobre a organização dita a forma como eles encaram o seu trabalho e quais os comportamentos que decidem adotar diariamente; dessa forma, clima organizacional é considerado como os significados e as perceções globais que um trabalhador tem do seu local de trabalho, baseado nas experiências vivenciadas (SCHNEIDER; EHRHART; MACEY, 2013).

Por ser baseado em perceções, quando os trabalhadores têm uma perceção positiva do ambiente que vivenciam na organização, i.e., quando avaliam, positivamente, as práticas, políticas e procedimentos organizacionais, encaram seu trabalho, positivamente, e tendem a sentir-se mais satisfeitos com ele (AHMAD; JASIMUDDIN; KEE, 2018). Consequentemente, propõe-se a seguinte hipótese:

H6: O Clima Organizacional está relacionado, positivamente, com a Satisfação no Trabalho.

De forma a inovar e a superar os concorrentes, as organizações vêm-se obrigadas a reestruturar seus sistemas e suas políticas, tornando-se, assim, mais rigorosas para com seus trabalhadores (SONNENTAG; FRITZ, 2015), influenciando seu estado de saúde, à proporção que se veem, constantemente, em situações estressantes (RANA; MUNIR, 2011).

Consequentemente, Stress no trabalho é definido como a reação que o corpo tem quando é exposto a estímulos do ambiente (RANA; MUNIR, 2011), em que o trabalhador vê-se obrigado a "[...] desviar do seu funcionamento normal como resultado das exigências relacionadas ao seu trabalho." (PARKER; DECOTIIS, 1983, p. 165, tradução nossa).

A relação negativa entre Stress e Satisfação no trabalho tem sido amplamente corroborada por diversos autores, na medida em que concordam que, efetivamente, o stress provoca problemas de saúde e mal-estar e ainda contribui para problemas organizacionais, como a baixa motivação, o baixo desempenho e a insatisfação no trabalho (CAVANAUGH et al., 2000). Desse modo, propõe-se a seguinte hipótese:

H7: O Stress está relacionado, negativamente, com a Satisfação no Trabalho.

Embora seja importante no local de trabalho fazer uso de programas de incentivos não monetários, é igualmente importante remunerar os trabalhadores por seu trabalho, pois eles precisam, efetivamente, de verbas para se sustentarem (JUDGE et al., 2010) uid=3992c099-6777-42c0-81d4-23c6aa5e31be"]\}], "mendeley": \{“formattedCitation":"(Appelbaum \& Kamal, 2000; T. Judge, Piccolo, Podsakoff, Shaw, \& Rich, 2010.

Dessa maneira, entende-se por salário, e segundo o artigo $258^{\circ}$ do código de trabalho (Lei $n^{\circ} 1$, de 20 de março de 2018) como a retribuição a que os trabalhadores têm direito em contrapartida de seu trabalho (PORTUGAL, 2018a, p.102) e, segundo o artigo $276^{\circ}$, do Código de Trabalho (Lei $n^{\circ} 1$, de 20 de março de 2018) sendo satisfeita em dinheiro (PORTUGAL, 2018b, p. 107).

As remunerações representam uma forma de as organizações comunicarem aos trabalhadores o seu valor para elas, assim e de acordo com Coning, Rothmann e Stander (2019), quando as organizações pagam salários justos a seus trabalhadores, estes retribuem com maior produtividade e com níveis elevados de comprometimento e de satisfação. Assim, propõe-se a seguinte hipótese:

H8: O salário está relacionado, positivamente, com a Satisfação no Trabalho.

As práticas massivas de downsizing e as mudanças repentinas dos mercados abalaram a moral e a confiança dos trabalhadores e, dessa forma, a inclusão de uma dimensão de espiritualidade no trabalho é importante de forma a recuperar o que, anteriormente, foi perdido (AFSAR; REHMAN, 2015)alignment with organizational values, sense of contribution to society, opportunities for inner life. 
Assim sendo, o equilíbrio emocional e a paz interior são uma dimensão desenvolvida por Joelle e Coelho (2017) integrada no conceito de espiritualidade no trabalho, que está " [...] associado a aspetos da vida de um indivíduo com a vida profissional, procurando o equilíbrio certo para assegurar o equilíbrio emocional e a paz interior." (JOELLE; COELHO, 2017, p. 5, tradução nossa).

A espiritualidade no trabalho está intimamente relacionada com a satisfação no trabalho, pois aumenta a qualidade de vida de seus colaboradores, tanto pessoal como profissional (AFSAR; REHMAN, 2015)voluminous data, and higher data rate are pushing to rethink the current generation of the cellular mobile communication. The next or fifth generation (5G; dessa forma, conforme Joelle e Coelho (2017) concluíram, o equilíbrio emocional e a paz interior introduziram um maior equilíbrio entre as dimensões de espiritualidade e uma maior coerência ao conceito, assume-se que, também, haverá uma relação positiva com a satisfação no trabalho. Consequentemente, propõe-se a seguinte hipótese:

H9: O Equilíbrio Emocional e a Paz Interior estão relacionados, positivamente, com a Satisfação no Trabalho.

\subsection{CONSEQUENTES DA SATISFA- ÇÃO COM O TRABALHO}

Satisfação com a vida é uma das dimensões do bem-estar subjetivo que recai na sua vertente cognitiva; assim, refere-se a um julgamento global da qualidade de vida de uma pessoa (DIENER et al., 1985).

Como as pessoas passam a maior parte do seu dia e da sua vida a trabalhar e, cada vez mais, existem pressões e tensões no local de trabalho, provocadas pelas incertezas do mercado, é importante compreender os determinantes que levam a que os trabalhadores sintam satisfação na sua vida, com o intuito de aumentá-la. Dessa forma, e segundo Fritzsche e Parrish, (2005, p. 195, tradução nossa) "[...] os trabalhadores que estão satisfeitos no traba- lho também tendem a ter vidas mais felizes e saudáveis" e assim é passível afirmar-se que a satisfação no trabalho tem impacto na vida em geral de um colaborador (BRIEF; WEISS, 2002)with the decades that followed up to the 1990s not being particularly fertile. Whereas job satisfaction generally continues to be loosely but not carefully thought of and measured as an affective state, critical work in the 1990s has raised serious questions about the affective status of job satisfaction in terms of its causes as well as its definition and measurement. Recent research has focused on the production of moods and emotions at work, with an emphasis, at least conceptually, on stressful events, leaders, work groups, physical settings, and rewards/punishment. Other recent research has addressed the consequences of workers' feelings, in particular, a variety of performance outcomes (e.g., helping behaviors and creativity de forma que se assume uma relação positiva entre as duas variáveis (UNANUE et al., 2017). Posto isso, propõe-se a seguinte hipótese:

H10: A Satisfação no Trabalho está relacionada, positivamente, com a Satisfação com a Vida.

A forma como uma empresa consegue atingir o sucesso no mercado competitivo é por meio da conquista de clientes (KOTLER; ARMSTRONG, 2018), e como já foi abordado, o foco nos trabalhadores é fundamental para que isso aconteça, pois são eles que asseguram um melhor atendimento ao cliente (LINDON et al., 2004).

A conquista de clientes torna-se, assim, possível quando as organizações implementam uma orientação para o cliente na cultura da organização que visa moldar as atitudes e os comportamentos dos trabalhadores em função do bom atendimento ao cliente (CHOI; JOUNG, 2017). Dessa forma, denominaremos orientação para o cliente como o "[...] grau em que os trabalhadores praticam o conceito de marketing, tentando ajudar os seus clientes a tomar decisões de compra que satisfaçam as necessidades do cliente." (SAXE; WEITZ, 1982, p. 344, tradução nossa).

Essa cultura orientada para o cliente só é 
possível de ser implementada no quotidiano dos trabalhadores por meio de políticas de recursos humanos que lhes proporcionam um elevado grau de satisfação com seus trabalhos, pois um trabalhador satisfeito, que sente preenchidas as suas necessidades, torna-se mais predisposto a criar bons relacionamentos com os clientes e a adotar comportamentos que visem satisfazer as necessidades deles (LEE et al., 2013). Consequentemente, propõe-se a seguinte hipótese:

H11: A Satisfação no Trabalho está relacionada, positivamente, com a Orientação para o Cliente.

A importância do comprometimento organizacional partiu da ideia de que uma empresa só alcança o sucesso se os seus trabalhadores estiverem comprometidos, se quiserem permanecer e trabalhar eficazmente e se quiserem contribuir com a sua produtividade para concretizar os objetivos organizacionais (BUDIHARDJO, 2013).

Dessa forma, comprometimento afetivo, uma das três dimensões de comprometimento organizacional mencionadas por Meyer e Allen (1991), refere-se ao desejo do trabalhador em permanecer na organização, ao seu envolvimento emocional, à sua identificação com os valores, visão e missão da empresa e à sua ligação com ela (MEYER; ALLEN, 1991).

Um colaborador que está, emocionalmente, comprometido com a organização tem uma maior predisposição para ser mais cooperativo e motivado a executar as suas tarefas (REGO; CUNHA, 2008); no entanto, esse laço efetivo só é possível se o trabalhador se sentir satisfeito com seu trabalho (BUDIHARDJO, 2013)t.au=Lee $\% 2 \mathrm{C}+$ Yong-Ki\&rft.date $=2005-01-01 \&$ rft.pub $=$ Elsevier+Ltd\&rft.i","ISSN":"02784319","abstract":'The service orientation program developed for restaurant employees can be a competitive advantage for a restaurant operation. The purpose of this study is to examine the relationship between employee service orientation (customer focus, organizational support, and service under pressure i.e., o sentimento de pertença do trabalhador resulta do sentimento de felicida- de e de satisfação que obtem de seu trabalho, quando ele vê as suas necessidades satisfeitas (MEYER; ALLEN, 1991). Consequentemente, propõe-se a seguinte hipótese:

H12: A Satisfação no Trabalho está relacionada, positivamente, com o Comprometimento Afetivo Organizacional.

Tal como o comprometimento, a lealdade tem um papel importante no contexto organizacional, pois é considerada um fator essencial para o desempenho das organizações bem como para aprimorar a satisfação e a lealdade do cliente (YEE; YEUNG; CHENG, 2010)we examine the relationships among employee loyalty, service quality, customer satisfaction, customer loyalty and firm profitability, and the contextual factors influencing these relationships. We developed a research model grounded in the service-profit chain notion of Heskett et al. (1994.

A lealdade é definida, geralmente, como sendo "[...] o sentimento de vinculação de um trabalhador à sua organização" (YEE; YEUNG; CHENG, 2010, p. 113, tradução nossa)we examine the of Heskett et al. (1994 e o desejo de permanecer a trabalhar nela (DAVIS-BLAKE; BROSCHAK; GEORGE, 2003).

O comprometimento e a lealdade partilham de bases teóricas semelhantes, pois ambos partem do sentimento de vinculação e envolvimento de um trabalhador com a sua empresa, tornando, assim, a ligação, entre ambos os conceitos, forte (SOLÍS; MONROY, 2015). Os sentimentos de pertença que podem ser sentidos por um trabalhador e os esforços que estão dispostos a investir para servir a organização são fruto da satisfação que sentem ao realizar seu trabalho (BUDIHARDJO, 2013), pois um trabalhador que está satisfeito com seu trabalho não procura a partida por outro local de trabalho, sendo assim e segundo Chang, Chiu e Chen (2010), esses trabalhadores produzem sentimentos de lealdade com sua organização. Como resultado, propõem-se as seguintes hipóteses: H13: O Comprometimento Afetivo Organizacional está relacionado, positivamente, com a Lealdade do trabalhador. 
H14: A Satisfação no Trabalho está relacionada, positivamente, com a Lealdade do trabalhador.

Uma empresa para destacar-se, no presente século, terá de fazer um bom uso dos seus recursos humanos, nomeadamente, encontrar sistemas nos quais os colaboradores sintam orgulho na organização pela qual trabalham, pois, quando isso acontece, predispõem-se a trabalhar mais para que ela sobreviva e prospere (NG; YAM; AGUINIS, 2018).

Por orgulho, consideramos o sentimento que os trabalhadores nutrem pela organização pela qual trabalham (KRAEMER; GOUTHIER; HEIDENREICH, 2017)the influence of pride in personal performance (PP.

Cada vez mais, autores estão mostrando satisfação no trabalho com o orgulho que um trabalhador sente por sua organização, pois, de acordo com Arnett, Laverie e McLane (2002), o sentimento de orgulho é baseado nas avaliações pessoais e sociais de cada trabalhador, e que a vertente "trabalho" é incluída nos critérios a serem avaliados, de maneira que, quando esses se sentem satisfeitos com o seu trabalho, avaliam, positivamente, a vertente "trabalho" desenvolvendo um orgulho em pertencer à organização pela qual trabalham, i.e., baseiam as suas emoções na avaliação do grau de satisfação que têm do seu trabalho. Pelo exposto, propõem-se a seguinte hipótese:

H15: A Satisfação no Trabalho está relacionada, positivamente, com o Orgulho na Organização.

Devido às incertezas do mercado e ao aumento da concorrência, as organizações passam a exigir aos seus recursos humanos mais inovação, criatividade e proatividade (FAY; HÜTTGES, 2016).

Segundo Crant (2000), iniciativa pessoal é uma das quatro dimensões integrantes do conceito de comportamento proativo, sendo que ela se refere a um síndrome comportamental de trabalho, baseada em ações autoiniciativas, proativas, persistentes em que os trabalhadores vão além do que lhes é competido, de modo a superar as dificuldades encontradas, prevendo as futuras e engendrando planos para superá-las (MENSMANN; FRESE, 2019).

Assim sendo, são esses tipos de trabalhadores que se tornam essenciais para a eficácia e a sobrevivência da organização (FRESE et al., 1997), de forma que é necessário compreender o que uma organização poderá fazer para que seus trabalhadores adotem esse tipo de comportamento em seu quotidiano. Segundo Stroppa e Spieß (2011), os trabalhadores sentem-se mais propensos a adotar comportamentos de iniciativa pessoal quando se sentem satisfeitos e motivados em seu trabalho, pois o gosto pelo ele leva-os a quererem ajudar a organização a ultrapassar todas as dificuldades encontradas. Também foi estudado anteriormente por Jauhari, Singh e Kumar (2017)http://www.mendeley.com/ documents/?uuid=4aef7489-9441-4902-9cd5-ebee9f6c4fa9"]\}],"mendeley": \{“formattedCitation":"'(Jauhari, Singh, \& Kumar, 2017 que esses trabalhadores que são dotados de iniciativa pessoal são os que têm uma maior predisposição para satisfazer o cliente; pois, como procuram maneiras para superar os problemas de forma a atingir os objetivos organizacionais, garantem sempre soluções para seus clientes. Como resultado, propõem-se as seguintes hipóteses:

H16: A Satisfação no Trabalho está relacionada, positivamente, com a Iniciativa Pessoal.

H17: A Iniciativa Pessoal está relacionada, positivamente, com a Orientação para o Cliente.

\section{MÉTODO}

O modelo conceptual, representado na figura 1, mostra as hipóteses identificadas anteriormente.

Figura 1 - Modelo conceptual 


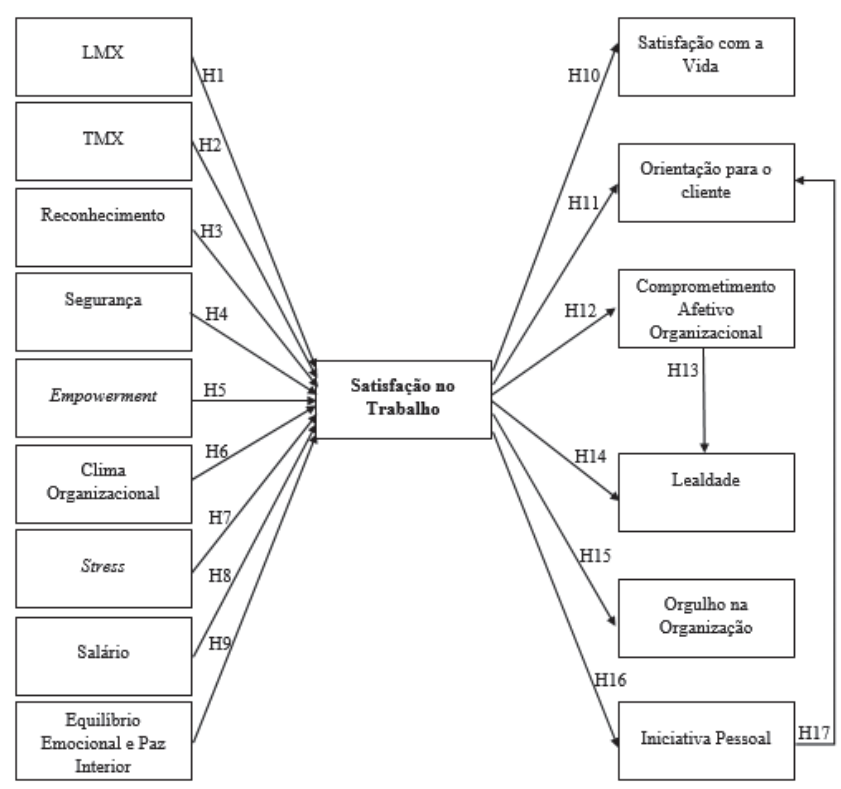

Fonte: elaboração própria (2019).

\section{ANÁLISE ESTATÍSTICA}

Para responder aos objetivos presentes neste artigo, realizou-se um estudo cross-section, por meio de um método não probabilístico por conveniência, utilizando um questionário de caráter fechado, elaborado por escalas de autores conceituados respondidas segundo uma escala de Likert de 7 pontos. O questionário em que foi elaborado online através do google for$m s$ e foi igualmente divulgado online por meio das redes sociais a trabalhadores em território português de qualquer sector de trabalho.

\subsection{CARACTERIZAÇÃO DAAMOSTRA}

A amostra é constituída por 418 trabalhadores portugueses em que $51,67 \%$ são do sexo feminino e $48,33 \%$ do masculino. A grande parte, assumindo uma escala entre 18 e mais de 56 anos, encontra-se entre a faixa etária dos 18 aos $45 \operatorname{anos}(82,3 \%)$ e é maioritariamente solteiro $(57,89 \%)$. O nível de escolaridade dessa amostra é mais disperso visto que $38,03 \%$ concluíram o ensino superior e $33,73 \%$ o ensino secundário. A grande parte dessa amostra trabalha no sector terciário $(80,38 \%)$, como funcionários de $2^{\circ}$
$(49,28 \%)$ e $1^{\circ}(36,60 \%)$ ordem, há menos de 10 anos $(78,23 \%)$, exercendo as atuais funções há menos de 5 anos $(59,09 \%)$. Por fim, as organizações, nas quais essa amostra trabalha, são constituídas, maioritariamente, por menos de 250 colaboradores $(79,67 \%)$ e estão em atividades empresariais a menos de 50 anos (90,19\%).

\subsection{MÉTRICAS}

O questionário utilizado é de caráter fechado, sendo respondido segundo a escala intervalar de Likert de 7 pontos, em que 1 corresponde a discordo fortemente; 7 corresponde a concordo fortemente, com exceção do item $n^{\circ}$ 4 da escala do salário em que 1 é definitivamente não e 7 definitivamente sim, e no item $n^{0} 5$ na escala da satisfação no trabalho em que 1 é muito insatisfeito e 7 muito satisfeito.

Para medir a satisfação no trabalho, utilizou-se a escala de Netemeyer et al. (1997), na qual se acrescentaram 2 itens da escala de Brayfield e Rothe (1951), utilizados, posteriormente, por Judge, Bono e Locke (2000).

Relativamente aos antecedentes da satisfação no trabalho, para medir o LMX utilizou-se a escala de Graen e Uhl-Bien (1995) 
e para medir o TMX, utilizou-se a escala de Seers, Petty e Cashman (1995). Relativamente ao reconhecimento, utilizou-se a escala de Matzler, Fuchs, e Schubert (2004), em que se acrescentou 1 item da escala de Spector (1985), revertendo para a positiva. Para medir a segurança, utilizou-se a escala, revertendo-a para a positiva, de Vander Elst, De Witte e De Cuyper (2014) e, para medir o empowerment, utilizou-se a escala de Gounaris (2008)the extant literature reports that the number of companies practicing marketing internally is disproportionate small compared to the number of companies trying to adopt the market orientation concept Hence, the purpose of this paper is to offer a preliminary insight regarding the antecedents of practicing marketing internally. Design/ methodology/approach - To do this, data were collected from 583 first-line personnel from 29 five and four stars hotels in Greece through personal interviews in order to investigate the impact of company culture and internal-market orientation (IMO. Em relação ao clima organizacional, utilizou-se a escala de Wangenheim, Evanschitzky e Wunderlich (2007) e, para medir o stress, utilizou-se escala de House e Rizzo (1972)designed to measure various internal organizational and managerial practices. Eight of 19 scales were validated against eight criterion variables measuring role stress, satisfaction, and leader behavior. An adaptation of the Campbell and Fiske (1959. Para medir os salários, recorreu-se à escala de Christen, Iyer e Soberman (2006) e, finalmente, relativamente ao equilíbrio emocional e paz interior, utilizou-se a escala de Joelle e Coelho (2017).

Em relação aos consequentes da satisfação no trabalho, para medir a satisfação com a vida, utilizou-se a escala de Diener et al. (1985) e para medir a orientação para o cliente, utilizou-se a escala de Korschun, Bhattacharya e Swain (2014). Relativamente ao comprometimento afetivo organizacional, utilizou-se a escala de Meyer, Allen e Smith (1993)continuance, and nodb3156248"] \}],"mendeley": \{“formattedCitation":"(Meyer, Allen, \& Smith, 1993, para a lealdade, utilizou-se a escala de Davis-Blake,
Broschak e George (2003) e, para medir o orgulho na organização, recorreu-se à escala de Helm (2013)it Pride: documents/?uuid=0501aa5e-ef17-4ab9-b74b-1 ee498d57b73"] \}],"mendeley": \{“formattedCitation":"(Helm, 2013. Para finalizar, e medir a iniciativa pessoal, recorreu-se à escala de Frese et al. (1997).

\subsection{VALIDADE}

Para avaliar a qualidade das escalas utilizadas e do ajustamento do modelo de medidas, foi realizada uma análise fatorial confirmatória com suporte do software estatístico IBM SPSS AMOS v.25.

O modelo de medidas final apresenta um bom ajustamento, pois obteve os seguintes valores: Incremental fit índex $=0,919$; TuckerLewis índex $=0,912$; comparative fit index $=$ 0,919 ; root mean square error of approximation $=0,053 ; \mathrm{X} 2 / \mathrm{DF}=2,183$.

Para análise da fiabilidade das variáveis, analisou-se o composite reliability (CR), em que todos os valores foram superiores a 0,7 , e o average variance extracted (AVE), em que todos os valores foram superiores a 0,5 . Dessa forma, indo ao encontro das recomendações da literatura, pode-se afirmar que as variáveis são fiáveis (HAIR et al., 2014). Relativamente à validade discriminante das variáveis, pode-se observar que as correlações ao quadrado entre as variáveis são inferiores às suas respetivas AVES, com exclusão da correlação entre as variáveis, comprometimento afetivo organizacional e lealdade e entre as variáveis, lealdade e orgulho na organização. Porém, testado um modelo alternativo de validade discriminante, sugerido por Fornell e Larcker (1981), em que é feito um modelo exclusivo para cada par de variáveis, fixando a correlação em 1, observa-se que os valores obtidos são, significativamente, superiores quando a correlação está fixada em 1, comprovando, assim, validade discriminante (tabela 1). 
Tabela 1- Desvio-padrão (DP), matriz de correlações, Alpha de Cronbach (sublinhado), Variância Média Extraida (AVE) e Fiabilidade Compósita (CR)

\begin{tabular}{|c|c|c|c|c|c|c|c|c|c|c|c|c|c|c|c|c|c|c|c|}
\hline & DP & LMX & TMX & REC & SEG & EMP & Clio & Stress & SAL & EEPI & ST & SV & OC & CAO & LEAL & ORG & IP & AVE & CR \\
\hline LMX & 1,546 & 0,946 & & & & & & & & & & & & & & & & 0,749 & 0,947 \\
\hline TMX & 1,282 & 0,527 & 0,939 & & & & & & & & & & & & & & & 0,729 & 0,941 \\
\hline REC & 1,831 & 0,687 & 0,418 & 0,947 & & & & & & & & & & & & & & 0,859 & 0,948 \\
\hline SEG & 1,592 & 0,567 & 0,608 & 0,527 & 0,940 & & & & & & & & & & & & & 0,796 & 0,940 \\
\hline EMP & 1,567 & 0,712 & 0,536 & 0,643 & 0,622 & 0,947 & & & & & & & & & & & & 0,821 & 0,948 \\
\hline CliO & 1,330 & 0,694 & 0,593 & 0,653 & 0,596 & 0,707 & 0,879 & & & & & & & & & & & 0,599 & 0,881 \\
\hline Stress & 1,378 & $-0,238$ & $-0,080$ & $-0,270$ & $-0,180$ & $-0,230$ & $-0,237$ & 0,898 & & & & & & & & & & 0,598 & 0,899 \\
\hline SAL & 1,755 & 0,480 & 0,270 & 0,701 & 0,519 & 0,497 & 0,545 & $-0,139$ & 0,814 & & & & & & & & & 0,536 & 0,820 \\
\hline EEPI & 1,837 & 0,541 & 0,441 & 0,556 & 0,536 & 0,535 & 0,586 & $-0,282$ & 0,518 & 0,967 & & & & & & & & 0,881 & 0,967 \\
\hline ST & 1,654 & 0,650 & 0,521 & 0,641 & 0,678 & 0,655 & 0,691 & $-0,315$ & 0,621 & 0,761 & 0,938 & & & & & & & 0,800 & 0,941 \\
\hline SV & 1,576 & 0,488 & 0,473 & 0,510 & 0,598 & 0,552 & 0,536 & $-0,152$ & 0,571 & 0,613 & 0,681 & 0,922 & & & & & & 0,720 & 0,927 \\
\hline OC & 1,256 & 0,439 & 0,732 & 0,308 & 0,468 & 0,448 & 0,573 & 0,039 & 0,222 & 0,386 & 0,452 & 0,434 & 0,944 & & & & & 0,815 & 0,964 \\
\hline CAO & 1,586 & 0,607 & 0,549 & 0,606 & 0,618 & 0,611 & 0,687 & $-0,232$ & 0,563 & 0,729 & 0,786 & 0,611 & 0,474 & 0,921 & & & & 0,753 & 0,924 \\
\hline LEAL & 1,424 & 0,623 & 0,603 & 0,605 & 0,683 & 0,651 & 0,718 & $-0,277$ & 0,574 & 0,767 & 0,826 & 0,651 & 0,533 & 0,938 & 0,862 & & & 0,697 & 0,873 \\
\hline ORG & 1,806 & 0,603 & 0,494 & 0,601 & 0,601 & 0,606 & 0,643 & $-0,254$ & 0,578 & 0,738 & 0,808 & 0,598 & 0,447 & 0,847 & 0,932 & 0,978 & 0,938 & 0,978 \\
\hline IP & 1,351 & 0,468 & 0,720 & 0,362 & 0,498 & 0,520 & 0,607 & $-0,018$ & 0,294 & 0,504 & 0,509 & 0,475 & 0,789 & 0,550 & 0,614 & 0,521 & 0.961 & 0,804 & 0,961 \\
\hline
\end{tabular}

Nota: LMX - leader-member exchange; TMX - team-member exchange; REC - reconhecimento; SEG- segurança, EMP - empowerment; CliO - clima organizacional; SAL - salários; EEPI - equilíbrio emocional e paz interior; ST - satisfação no trabalho; SV - satisfação com a vida; OC - orientação para o cliente; CAO - comprometimento afetivo organizacional; LEAL - lealdade; ORG - orgulho; IP - Iniciativa pessoal.

Fonte: elaboração própria (2019).

\section{RESULTADOS}

Para testar as hipóteses, recorre-se à análise do modelo das equações estruturais, aplicando, novamente, o software estatístico IBM SPSS AMOS v.25.

Analisando-o, observa-se que esse apresenta um bom ajustamento, pois obteve os seguintes valores: Incremental fit índex $=0,902$; Tucker-Lewis índex $=0,897$; comparative fit index $=0,902$; root mean square error of approximation $=0,058 ; \mathrm{X} 2 / \mathrm{DF}=2,388$.

É passível de se comprovar os resultados relativamente às hipóteses propostas na tabela 2 .

\begin{tabular}{|c|c|c|c|c|}
\hline $\mathrm{H}$ & Relaçã & & Estimate & $\mathrm{P}$ \\
\hline $\mathrm{H} 1$ & LMX & $\rightarrow \mathrm{ST}$ & 0,082 & 0,053 \\
\hline $\mathrm{H} 2$ & TMX & $\rightarrow \mathrm{ST}$ & 0,086 & 0,017 \\
\hline $\mathrm{H} 3$ & REC & $\rightarrow \mathrm{ST}$ & $-0,021$ & 0,655 \\
\hline $\mathrm{H} 4$ & SEG & $\rightarrow \mathrm{ST}$ & 0,171 & $* * *$ \\
\hline $\mathrm{H} 5$ & EMP & $\rightarrow \mathrm{ST}$ & 0,084 & 0,047 \\
\hline H6 & $\mathrm{CliO}$ & $\rightarrow$ ST & 0,129 & 0,006 \\
\hline $\mathrm{H} 7$ & Stress & $\rightarrow \mathrm{ST}$ & $-0,065$ & 0,016 \\
\hline $\mathrm{H} 8$ & SAL & $\rightarrow$ ST & 0,181 & $* * *$ \\
\hline H9 & EEPI & $\rightarrow \mathrm{ST}$ & 0,409 & $* * *$ \\
\hline H10 & ST & $\rightarrow \mathrm{SV}$ & 0,72 & $* * *$ \\
\hline H11 & ST & $\rightarrow \mathrm{OC}$ & 0,074 & 0,075 \\
\hline H12 & ST & $\rightarrow \mathrm{CAO}$ & 0,85 & $* * *$ \\
\hline H13 & $\mathrm{CAO}$ & $\rightarrow$ LEAL & 0,61 & $* * *$ \\
\hline H14 & ST & $\rightarrow$ LEAL & 0,38 & $* * *$ \\
\hline H15 & ST & $\rightarrow$ ORG & 0,867 & $* * *$ \\
\hline H16 & ST & $\rightarrow$ IP & 0,58 & $* * *$ \\
\hline H17 & IP & $\rightarrow \mathrm{OC}$ & 0,746 & $* * *$ \\
\hline
\end{tabular}

Fonte: elaboração própria (2019). 
Relativamente ao LMX (SRW=0,082; $\mathrm{p}<0,1)$ e ao TMX (SRW=0,086; $\mathrm{p}<0,05)$ consegue-se observar que há um impacto positivo na Satisfação no Trabalho, suportando H1 e $\mathrm{H} 2$ respetivamente. Como foi comprovado em estudos anteriores, aqui também se corrobora que os bons relacionamentos entre o líder e o liderado partem da base de uma parceria madura (GRAEN; UHL-BIEN, 1995) em que existem confiança, respeito e lealdade que proporcionam mais benefícios para os trabalhadores (MORROW et al., 2005), e que os bons relacionamentos com os colegas de trabalho resultam em entreajuda, maior comunicação, troca de informações e feedback (DUAN; XU; FRAZIER, 2018) sendo que, no fim, todos esses fatores proporcionam experiências de trabalho mais gratificantes que, em última análise, aumentam a satisfação no trabalho (BHAL; GULATI; ANSARI, 2009; BANKS et al., 2014).

Contrariamente ao esperado e ao corroborado nos estudos de Appelbaum e Kamal (2000)tempm \& Kamal, 2000 e Alias et al. (2018), verifica-se que o Reconhecimento não tem um impacto significativo na satisfação no trabalho ( $S R W=-0,021 ; p>0,1)$, não suportando H3. Isso poderá ser pelo fato de, relativamente a esta amostra, haver muito pouca atenção por parte dos empregadores quanto ao reconhecimento como sendo um fator importante para o bom desenvolvimento organizacional.

Comparativamente às variáveis Segurança no Trabalho ( $S R W=0,171 ; p<0,01)$ e Salário (SRW=0,181; $\mathrm{p}<0,01)$, observa-se que elas têm um impacto positivo na Satisfação no Trabalho, o que suporta $\mathrm{H} 4$ e $\mathrm{H} 8$ respetivamente. As incertezas do mercado enfatizam as preocupações dos trabalhadores; dessa forma, comprova-se que, quando as organizações comunicam a seus trabalhadores que tanto eles como seus postos de trabalho são valorizados, eles detêm maiores níveis de satisfação com o trabalho (GAZIOGLU; TANSEL， 2006; CONING; ROTHMANN; STANDER, 2019; RICHTER; NÄSWALL, 2019)quits tion: Research regarding subjective well-being (including life satisfaction and domain-specific satisfaction.
No que toca ao Empowerment ( $S R W=0,084 ; p<0,05)$ e ao Clima Organizacional (SRW=0,129; $\mathrm{p}<0,01)$, é suscetível de se observar que há um impacto positivo na Satisfação no Trabalho, o que suporta $\mathrm{H} 5$ e H6, respetivamente. Igualmente, corroborando estudos anteriores, quando os trabalhadores são envolvidos nos processos de tomada de decisões, sentem-se honrados pela confiança depositada e, quando se identificam com as práticas, políticas e procedimentos das quais a sua empresa se mune, avaliam as experiências no trabalho positivamente, e, assim sendo, sentem-se mais satisfeitos no trabalho (UGBORO; OBENG, 2000; WANGENHEIM; EVANSCHITZKY; WUNDERLICH, 2007; KIM; FERNANDEZ, 2017; AHMAD; JASIMUDDIN; KEE, 2018) many topior research has used, it investigates whether or not the level of customer contact is a determinant of the existence or the intensity of the employee-customer satisfaction link. Analysis of dyadic data from 53,645 customers and 1659 employees across 99 outlets of a large German Do-It-Yourself (DIY.

Como era previsto, a variável Stress demonstrou ter um impacto negativo e significativo na satisfação no trabalho ( $\mathrm{SRW}=-0,065$; $\mathrm{p}<0,05$ ), corroborando, assim, $\mathrm{H} 7$ bem como o autor Cavanaugh et al. (2000) que previram que elevados níveis de stress contribuem para que os trabalhadores se sintam insatisfeitos com seus trabalhos, pois experienciam ambientes de trabalho em que prevalece o mal-estar, levando a que não consigam tirar satisfação do trabalho.

Em contrapartida, o Equilíbrio Emocional e a Paz Interior têm um impacto positivo na satisfação no trabalho (SRW=0,409; $p<0,01$ ), o que corrobora H9. Dessa forma, este estudo, indo de encontro com o de Joelle e Coelho (2017), comprova que as organizações, ao abrirem espaço à espiritualidade, reconhecem a importância da felicidade e da paz interior e possibilitam uma maior harmonia e equilíbrio entre a vida pessoal e a profissional de um trabalhador, aumentando a sua satisfação no trabalho.

Respetivamente à Satisfação no Trabalho, essa variável demonstrou ter um impacto 
positivo na satisfação com a vida (SRW=0,72; $\mathrm{p}<0,01$ ), o que suporta H10. Essa relação positiva corrobora estudos prévios que argumentam que as experiências que um trabalhador retira de seu emprego transferem-se para o domínio da vida, na medida em que, quando ele obtém experiências profissionais positivas, considera a sua vida mais feliz e satisfatória (UNANUE et al., 2017).

A Satisfação no Trabalho também demonstrou ter uma relação positiva com a Orientação para o Cliente (SRW=0,074; $<<0,1)$, Comprometimento Afetivo Organizacional (SRW=0,85; $\mathrm{p}<0,01)$, lealdade $(\mathrm{SRW}=0,38$; $\mathrm{p}<0,01$ ), Orgulho na Organização (SRW= 0,$867 ; p<0,01$ ) e Iniciativa Pessoal ( $S R W=0,58$; $\mathrm{p}<0,01$ ), suportando respetivamente, H11, H12, H14, H15 e H16. Dessa forma, corroboram os autores que, anteriormente, provaram que, quando uma empresa consegue trazer satisfação a seus trabalhadores, consegue, com maior facilidade, implementar neles uma orientação para o cliente à medida que eles, ao se sentirem motivados a fazer o seu trabalhado, têm comportamentos que visam valorizar mais os seus clientes (CHOI; JOUNG, 2017); faz que eles desenvolvam sentimentos de pertença com a organização sendo-lhes mais leais, o que significa que farão tudo para permanecer nela, sendo mais cooperativos, esforçados e motivados (REGO; CUNHA, 2008); que eles se identifiquem com a missão e os valores da organização, o que faz que desenvolvam um sentimento de orgulho em fazer parte dela (ARNETT; LAVERIE; MCLANE, 2002); e com que sejam mais proativos e tenham iniciativa pessoal, de modo que desenvolvam planos tanto para superar dificuldades encontradas no momento, como para as futuras, de forma a concretizar os objetivos organizacionais e a ajudar a empresa a prosperar (MENSMANN; FRESE, 2019).

Também se observa que o Comprometimento Afetivo Organizacional tem um impacto positivo na Lealdade ( $S R W=0,61, p<0,01$ ), corroborando $\mathrm{H} 13$ e indo de encontro com estudos prévios que comprovam que um trabalhador que está comprometido com a organização é-lhe leal
(SOLÍS; MONROY, 2015) e que a Iniciativa Pessoal tem um impacto positivo na orientação para o cliente $(\mathrm{SRW}=0,746 ; \mathrm{p}<0,01)$ corroborando $\mathrm{H} 17$ e a ideia de que somente os trabalhadores proativos e dotados de iniciativa pessoal têm uma maior predisposição para satisfazer os clientes (JAUHARI; SINGH; KUMAR, 2017) the authors of6c4fa9"]\}],"mendeley":\{“formattedCitation":"(Jauhari et al., 2017.

\section{CONCLUSÃO}

O objetivo central deste estudo é compreender melhor o que leva à satisfação no trabalho e de que maneira um trabalhador satisfeito responde perante a sua satisfação. Para se conseguir dar resposta ao proposto e testar as hipóteses de investigação, foi realizado um modelo de equações estruturais, que teve por base a amostra de 418 trabalhadores obtidos por meio de um questionário de um estudo cross-section.

Os resultados obtidos demonstraram efetivamente que existem muitos fatores que estão nas mãos das empresas que provocam satisfação no trabalho, sendo que as vantagens as quais as organizações conseguem ao se focarem na satisfação dos trabalhadores são consideráveis, pois os trabalhadores, estando satisfeitos, ficam mais orientados para os clientes, comprometidos, leais e focados em concretizar os objetivos da organização.

Este estudo visa a uma melhor compreensão por parte das organizações de que um maior foco nos seus recursos humanos, pois é uma estratégia inteligente devido aos seus resultados organizacionais positivos e vantajosos, que se podem traduzir em vantagem competitiva.

\section{CONTRIBUIÇÕES E LIMITAÇÕES}

\subsection{CONTRIBUIÇÕES TEÓRICAS}

Este estudo pretende contribuir para a construção de um modelo, com recurso à literatura, que analisa vasta e globalmente o concei- 
to de satisfação no trabalho, bem como analisa, simultaneamente, as causas e os efeitos de um trabalhador satisfeito, pois a maioria dos estudos foca-se em analisá-los separadamente; para o desenvolvimento de um estudo geral sobre satisfação que visa preencher a lacuna de estudos sobre o tema, pois agrupa um conjunto de variáveis que nunca foram estudadas em conjunto relativamente à satisfação no trabalho, permitindo a exploração em maior profundidade do tema e para o reforço da literatura sobre satisfação no trabalho, dando a conhecer a realidade mais recente dos trabalhadores relativamente aos fatores que consideram influenciadores da sua satisfação, e os resultados de estarem satisfeitos, já que o tema lida com as mudanças constantes do mercado que tornam imprescindível o seu estudo contínuo de modo a mantê-los atualizado.

\subsection{CONTRIBUIÇÕES PRÁTICAS}

Em um âmbito prático, este estudo esclarece às organizações que, efetivamente, existem muitos fatores em suas mãos que dão satisfação a seus trabalhadores, e que existem muitas vantagens organizacionais em manter os seus trabalhadores satisfeitos; pois, quando uma organização consegue que eles encarem o seu trabalho de maneira satisfatória, essas experiências positivas influenciam não só em sua vida pessoal, pois a torna mais feliz e satisfatória, como também em sua vida profissional, uma vez que ficam mais propensos a ter comportamentos que atendam às necessidades de seus clientes ao desenvolver laços efetivos com a organização; a identificar-se e serem leais a ela, ou seja, a trabalhar para responder aos objetivos propostos de maneira que o relacionamento entre ambas não acabe; a ter um mais orgulho em pertencer à organização; a defendê-la, e a envolver-se em iniciativas pessoais e proativas para resolver os problemas encontrados, ajudando a organização a prosperar.

Concluindo, é demonstrada a importância de um foco na satisfação dos trabalhadores e como estes poderão ser uma estratégia inteli- gente para alcançar uma vantagem competitiva, pois, ao estarem satisfeitos, estão mais predispostos a colaborar, eficientemente, nas estratégias organizacionais.

\subsection{LIMITAÇÕES E PES- QUISAS FUTURAS}

Esta pesquisa aponta como limitações a falta de tempo e de recursos, que faz que o estudo tenha de ser baseado em um estudo cross-section com uma amostra de 418 trabalhadores portugueses, obtida por meio de um método não probabilístico por conveniência, que não permite extrapolar os dados para a população. Dessa forma, estudos futuros deveriam considerar uma amostra mais expressiva conseguida por métodos de amostragem probabilísticos de forma a conseguirse generalizar os dados para a população.

As variáveis escolhidas como antecedentes e consequentes da satisfação no trabalho não são exaustáveis, pois se propõe que pesquisas futuras explorem outros antecedentes e consequentes, para contínua contribuição do preenchimento de lacunas sobre os estudos da temática.

\section{REFERÊNCIAS}

AFSAR, B.; REHMAN, M. The relationship between workplace spirituality and innovative work behavior: The mediating role of perceived person-organization fit. Journal of Management, Spirituality and Religion, v. 12, n. 4, p. 329-353, 2015.

AHMAD, K. Z. B.; JASIMUDDIN, S. M.; KEE, W. L. Organizational climate and job satisfaction: do employees' personalities matter? Management Decision, 2018.

AHMED, P. K.; RAFIQ, M. Internal Marketing: tools and Concepts for customer-focused management. [S.l.]: Butterworth-Heinemann, 2002.

ALBALOOSHI, A.; ALI, A.; AL-ANSI, A. The Effect of Job Loyalty, Management Performance and Rewards and Recognition on Prof- 
itability of Islamic and Conventional Banks: Evidence from UAE. International Business Management, v. 11, n. 3, p. 721-728, 2017.

ALEGRE, I.; MAS-MACHUCA, M.; BERBEGAL-MIRABENT, J. Antecedents of employee job satisfaction: Do they matter? Journal of Business Research, v. 69, n. 4, p. 1390-1395, 2016.

ALIAS, N. E. et al. The Effect of Payment, Recognition, Empowerment and Work-Life Balance on Job Satisfaction in the Malaysia's Oil and Gas Industry. International Journal of Academic Research in Business and Social Sciences, v. 8, n. 9, p. 639-656, 2018.

ALMEIDA, F. Introdução à gestão de organizações. 4. ed. Lisboa: Escolar Editora, 2016.

APPELBAUM, S. H.; KAMAL, R. An analysis of the utilization and effectiveness of non-financial incentives in small business. Journal of Management Development, v. 19, n. 9, p. 733-763, 2000.

ARISTOVNIK, A. et al. Excellence in public administration: job satisfaction as a factor of good administration. International Journal of Human Resources Development \& Management, v. 18, n. 1/2, p. 127-144, 2018.

ARNETT, D.; LAVERIE, D.; MCLANE, C. Using Job Satisfaction and Pride as internal-marketing tools. Cornell Hotel and Restaurant Administration Quarterly, p. 87-96, 2002.

BANKS, G. C. et al. What does team-member exchange bring to the party? a meta-analytic review of team and leader social exchange. Journal of Organizational Behavior, v. 35, p. 273-295, 2014.

BANSAL, H. S.; MENDELSON, M. B.; SHARMA, B. The impact of internal marketing activities on external marketing outcomes. Journal of Quality Management, v. 6, n. 1, p. 61-76, 2001.
BHAL, K. T.; GULATI, N.; ANSARI, M. A. Leader-member exchange and subordinate outcomes: test of a mediation model. Leadership \& Organization Development Journal, v. 30, n. 2, p. 106-125, 2009.

BRAYFIELD, A. H.; ROTHE, H. F. An index of job satisfaction. Journal of Applied Psychology, v. 35, n. 5, p. 307-311, 1951.

BRIEF, A. P.; WEISS, H. M. Organizational Behavior: Affect in the Workplace. Annual Review of Psychology, v. 53, n. 1, p. 279-307, 2002.

BUDIHARDJO, A. The Relationship Between Job Satisfaction, Affective Commitment, Organizational Learning Climate and Corporate Performance. Journal on Business Review, v. 2, n. 4, p. 58-64, 2013.

CAVANAUGH, M. A. et al. An empirical examination of self-reported work stress among U.S. managers. Journal of Applied Psychology, v. 85, n. 1, p. 65-74, 2000.

CHANG, C. C.; CHIU, C. M.; CHEN, C. A. The effect of TQM practices on employee satisfaction and loyalty in government. Total Quality Management and Business Excellence, v. 21, n. 12, p. 1299-1314, 2010.

CHOI, E. K.; JOUNG, H. W. Employee job satisfaction and customer-oriented behavior: A study of frontline employees in the foodservice industry. Journal of Human Resources in Hospitality and Tourism, v. 16, n. 3, p. 235251, 2017.

CHRISTEN, M.; IYER, G.; SOBERMAN, D. Job Satisfaction, Job Performance, and Effort: A Reexamination Using Agency Theory. Journal of Marketing, v. 70, p. 137-150, 2006.

CONING, J. A. de; ROTHMANN, S.; STANDER, M. W. Do wage and wage satisfaction compensate for the effects of a dissatisfying job on life satisfaction? SA. Journal of Industrial 
Psychology, v. 45, p. 1-11, 2019.

CRANT, J. M. Proactive behavior in organizations. Journal of Management, v. 26, n. 3, p. 435-462, 2000.

CUNHA, M. P. E. et al. Manual de gestão de pessoas e do capital humano. 2. ed. Lisboa: Edições Sílabo LDA, 2012.

DAVIS-BLAKE， A.; BROSCHAK， J. P.; GEORGE, E. Happy together? How using nonstandard workers affects exit, voice, and loyalty among standard employees. Academy of Management Journal, v. 46, n. 4, p. 475-485, 2003.

DAVY, J. A.; KINICKI, A. J.; SCHECK, C. L. A Test of Job Security's Direct and Mediated Effects on Withdrawal Cognitions. Journal of Organizational Behavior, v. 18, p. 323-349, 1997.

DIENER, E. Assessing Well-Being. USA: Springer, 2009.

DIENER, E. et al. The Satisfaction With Life Scale. Journal of Personality Assessment, v. 49, n. 1, p. 71-75, 1985.

DUAN, J.; XU, Y.; FRAZIER, M. L. Voice Climate, TMX, and Task Interdependence: A Team-Level Study. Small Group Research, p. $1-28,2018$.

FAY, D.; HÜTTGES, A. Drawbacks of Proactivity: Effects of Daily Proactivity on Daily Salivary Cortisol and Subjective WellBeing. Journal of Occupational Health Psychology, p. 1-48, 2016.

FISHER, R. J.; ACKERMAN, D. The Effects of Recognition and Group Need on Volunteerism: A Social Norm Perspective. Journal of Consumer Research, v. 25, 1998.

FORNELL, C.; LARCKER, D. F. Evaluating
Structural Equation Models with Unobservable Variables and Measurement Error. Journal of Marketing Research, v. 18, n. 1, p. 39-50, 1981.

FRESE, M. et al. The concept of personal initiative: Operationalization, reliability and validity in two German samples. Journal of Occupational and Organizational Psychology, v. 70, p. 139-161, 1997.

FRITZSCHE, B.; PARRISH, T. Theories and Research on Job Satisfaction. In Career Development and Couseling: Putting Theory and Research to Work. New Jersey: John Wiley \& Sons Ltd, 2005.

GAZIOGLU, S.; TANSEL, A. Job satisfaction in Britain: Individual and job related factors. Applied Economics, v. 38, p. 1163-1171, 2006.

GOUNARIS, S. Antecedents of internal marketing practice: Some preliminary empirical evidence. International Journal of Service Industry Management, v. 19, n. 3, p. 400-434, 2008.

GRAEN, G. B.; UHL-BIEN, M. Relationship-Based Approach to Leadership: Development of Leader-Member Exchange (LMX) Theory of Leadership over 25 Years: Applying a Multi-Level Multi-Domain Perspective. Leadership Quarterly, v. 6, p. 219-247, 1995.

HAIR, J. et al. Multivariate data analysis. Harlow: Pearson Education Limited, 2014.

HELM, S. A Matter of Reputation and Pride: Associations between Perceived External Reputation, Pride in Membership, Job Satisfaction and Turnover Intentions. British Journal of Management, v. 24, p. 542-556, 2013.

HERZBERG, F.; MAUSNER, B.; SNYDERMAN, B. The Motivation to Work. USA: Transaction Publisher, 1959.

HOUSE, R. J.; RIZZO, J. R. Toward the measurement of organizational practices: Scale de- 
velopment and validation. Journal of Applied Psychology, v. 56, n. 5, p. 388-396, 1972.

JAUHARI, H.; SINGH, S.; KUMAR, M. How does transformational leadership influence proactive customer service behavior of frontline service employees? Examining the mediating roles of psychological empowerment and affective commitment. Journal of Enterprise Information Management, v. 30, n. 1, p. 3048, 2017.

JIANG, L.; PROBST, T. M. The moderating effect of trust in management on consequences of job insecurity. Economic and Industrial Democracy, p. 1-25, 2016.

JOELLE, M.; COELHO, A. M. The impact of spirituality at work on workers' attitudes and individual performance. The International Journal of Human Resource Management, p. 1-25, 2017.

JUDGE, T. A.; BONO, J. E.; LOCKE, E. A. Personality and job satisfaction: The mediating role of job characteristics. Journal of Applied Psychology, v. 85, n. 2, p. 237-249, 2000.

JUDGE, T. A. et al. The relationship between pay and job satisfaction: A meta-analysis of the literature. Journal of Vocational Behavior, v. 77, p. 157-167, 2010.

KIM, S. Y.; FERNANDEZ, S. Employee Empowerment and Turnover Intention in the U.S. Federal Bureaucracy. American Review of Public Administration, v. 47, n. 1, p. 4-22, 2017.

KORSCHUN, D.; BHATTACHARYA, C. B; SWAIN, S. D. Corporate Social Responsibility, Customer Orientation, and the Job Performance of Frontline Employees. Journal of Marketing, v. 78, n. 3, p. 20-37, 2014.

KOTLER, P.; ARMSTRONG, G. Principles of Marketing. 17. ed. United Kingdom: Pearson Education Limited, 2018.
KRAEMER, T.; GOUTHIER, M. H. J.; HEIDENREICH, S. Proud to Stay or Too Proud to Stay? How Pride in Personal Performance Develops and How It Affects Turnover Intentions. Journal of Service Research, v. 20, n. 2, p. 152-170, 2017.

LEE, C. K. et al. The impact of CSR on casino employees' organizational trust, job satisfaction, and customer orientation: An empirical examination of responsible gambling strategies. International Journal of Hospitality Management, v. 33, p. 406-415, 2013.

LINDON, D. et al. Mercator XXI: Teoria e prática do marketing. 10. ed. Porto: Publicações Dom Quixote, 2004.

LOCKE, E. A. What is job satisfaction? Organizational Behavior and Human Performance, v. 4, p. 309-336, 1969.

MASLOW, A. H. A Theory of Human Motivation. Brooklyn: [s.n.], 1943.

MATZLER, K.; FUCHS, M.; SCHUBERT, A. Employee Satisfaction: Does Kano's Model Apply? Total Quality Management \& Business Excellence, v. 15, n. 9/10, p. 1179-1198, 2004.

MENSMANN, M.; FRESE, M. Who stays proactive after entrepreneurship training ? Need for cognition, personal initiative maintenance , and well - being. Journal of Organizational Behavior, v. 40, p. 20-37, 2019.

MESMER-MAGNUS, J. R.; DECHURCH, L. A. Information sharing and team performance: A meta-analysis. Journal of Applied Psychology, v. 94, n. 2, p. 535-546, 2009.

MEYER, J. P.; ALLEN, N. A Three-Component Conceptualization Of Organizational Commitment. Human Resource Management Review, v. 1, n. 1, p. 61-89, 1991. 
MEYER, J. P.; ALLEN, N. J.; SMITH, C. A. Commitment to Organizations and Occupations: Extension and Test of a Three-Component Conceptualization. Journal of Applied Psychology, v. 78, n. 4, p. 538-551, 1993.

MORROW, P. C. et al. The role of leader-member exchange in high turnover work environments. Journal of Managerial Psychology, v. 20, n. 8, p. 681-694, 2005.

NETEMEYER, R. G. et al. An investigation into the antecedents of organizational citizenship behaviors in a personal selling context. journal of marketing, v. 61, p. 85-98, 1997.

NG, T. W. H.; YAM, K. C.; AGUINIS, H. Employee perceptions of corporate social responsibility: Effects on pride, embeddedness, and turnover. Personnel Psychology, p. 1-31, 2018.

NGUYEN, A. N.; TAYLOR, J.; BRADLEY, S. Job autonomy and job satisfaction: new evidence. [S.l.]: Working paper 050. UK, 2003.

PARKER, D. F.; DECOTIIS, T. A. Organizational Determinants of Job Stress. Organizational Behavior and Human Performance, v. 32, p. 160-177, 1983.

PORTUGAL. Código de trabalho: artigo 258 $\mathrm{n}^{\circ} 1$, de 20 de março de 2018. Lisboa, 2018a. Disponível em: http://cite.gov.pt/asstscite/ downloads/legislacao/CT20032018.pdf. Acesso em: 12 nov. 2018.

PORTUGAL. Código de trabalho: artigo 276 $\mathrm{n}^{\circ} 1$, de 20 de março de 2018. Lisboa, 2018b. Disponível em: http://cite.gov.pt/asstscite/ downloads/legislacao/CT20032018.pdf. Acesso em: 12 nov. 2018.

PRADHAN, R. K.; DASH, S.; JENA, L. K. Do HR practices influence job satisfaction? examining the mediating role of employee engagement in indian public sector undertakings.
Global Business Review, v. 20, n. 1, p. 119132, 2019.

PRAJOGO, W. The relationship among emotional exhaustion, job satisfaction, performance, and intention to leave. Advances in Management \& Applied Economics, v. 9, n. 1, p. 21-29, 2019.

RANA, B.; MUNIR, K. Impact of stressors on the performance of employees. MPRA, Germany, paper 32729, 2011.

REGO, A.; CUNHA, M. P. Workplace spirituality and organizational commitment: An empirical study. Journal of Organizational Change Management, v. 21, n. 1, p. 53-75, 2008.

RICHTER, A.; NÄSWALL, K. Job insecurity and trust: Uncovering a mechanism linking job insecurity to well-being. Work and Stress, v. 33, n. 1, p. 22-40, 2019.

ROBBINS, S. P.; COUlTER, M. Administración. [S.l.]: Prentice Hall, 1996.

SARKER, M. A. R.; ASHRAFI, D. M. The relationship between internal marketing and employee job satisfaction: A study from retail shops in Bangladesh. Journal of Business and Retail Management Research, v. 12, n. 3, p. 149-159, 2018.

SAXE, R.; WEITZ, B. A. The SOCO Scale: A Measure of the Customer Orientation of Salespeople. Journal of Marketing Research, v. 19, n. 3, p. 343-351, 1982.

SCHNEIDER, B.; EHRHART, M. G.; MACEY, W. Organizational Climate and Culture. Annual Review of Psychology, v. 64, p. 361388, 2013.

SEERS, A.; PETTY, M. M.; CASHMAN, J. F. Team-Member Exchange Under Team and Traditional Management: A Naturally Occuring Quasi-Management. Group and Organiza- 
tion Management, v. 20, n. 1, p. 18-38, 1995.

SHEN, J.; TANG, C. How does training improve customer service quality? The roles of transfer of training and job satisfaction. European Management Journal, v. 36, p. 1-9, 2018.

SIBHOKO, O.; BAYAT, M. S. An investigation into employee job satisfaction and its impact on organizational effectiveness with special reference to the buffalo city college. Management Studies and Economic Systems, v. 4, n. 1, p. 71-78, 2019.

SINHA, D.; KUMAR, S. Comparative Study of Job Satisfaction of the Employees of Private \& Public Sector Banks. Journal of Management, v. 1, n. 1, p. 25-38, 2012.

SOLÍS, E.; MONROY, V. Between love and war: The effects of affective commitment in organizational politics and organizational performance. Journal of Organizational Culture, Communications and Conflict, v. 19, n. 2, p. 69-93, 2015.

SONNENTAG, S.; FRITZ, C. Recovery from job stress: The stressor-detachment model as an integrative framework. Journal of Organizational Behavior, v. 36, p. 72-103, 2015.

SPECTOR, P. Job satisfaction: application, assessment, causes and consequences. Thousand Oaks: SAGE Publications, Inc, 1997.

SPECTOR, P. E. Measurement of human service staff satisfaction: Development of the Job Satisfaction Survey. American Journal of Community Psychology, v. 13, n. 6, p. 693713, 1985.

STROPPA, C.; SPIEß, E. The role of social support and personal initiative. International Journal of Intercultural Relations, v. 35, p. 234-245, 2011.

UGBORO, I. O.; OBENG, K. Top management leadership, employee empowerment, job satisfaction, and customer satisfaction in TQM organizations: an empirical study. Journal of Quality Management, v. 5, p. 247-272, 2000.

UNANUE, w. et al. revisiting the Link between Job Satisfaction and Life satisfaction: the role of basic psychological needs. Frontiers in Psychology, v. 8, p. 1-17, 2017.

VANDER ELST, T.; DE WITTE, H.; DE CUYPER, N. The Job Insecurity Scale: A psychometric evaluation across five European countries. European Journal of Work and Organizational Psychology, v. 23, n. 3, p. 364-380, 2014.

WANGENHEIM, F. V.; EVANSCHITZKY, H.; WUNDERLICH, M. Does the employee-customer satisfaction link hold for all employee groups? Journal of Business Research, v. 60, p. 690-697, 2007.

WEISS, H. M. Deconstructing job satisfaction: Separating evaluations, beliefs and affective experiences. Human Resource Management Review, v. 12, p. 173-194, 2002.

YEE, R. W. Y.; YEUNG, A. C. L.; CHENG, T. C. E. An empirical study of employee loyalty, service quality and firm performance in the service industry. International Journal of Production Economics, v. 124, p. 109-120, 2010.

YIN, Y.; WANG, Y.; LU, Y. Why firms adopt empowerment practices and how such practices affect firm performance? A transaction cost-exchange perspective. Human Resource Management Review, p. 1-14, 2018.

YUKL, G.; GORDON, A.; TABER, T. A Hierarchical Taxonomy of Leadership Behavior: Integrating a Half Century of Behavior Research. Journal of Leadership and Organizational Studies, v. 9, n. 1, 2002. 